\title{
In-Vitro Antimalarial Resistance Pattern of Plasmodium Falciparum Infection Among Pregnant Women In Northern Nigeria
}

\author{
Yahaya Abdullahi Umar ${ }^{1}$, Idris Abdullahi Nasir ${ }^{2}$, Maryam Muhammad Aliyu ${ }^{1}$, \\ Amos Dangana ${ }^{2}$, Anthony Philip Vanstawa ${ }^{1}$ \\ ${ }^{1}$ Department of Biological Science, National Defense Academy, Kaduna State, Nigeria \\ ${ }^{2}$ Department of Medical Laboratory Services, University of Abuja Teaching Hospital Gwagwalada, \\ FCT Abuja, Nigeria
}

Corresponding Author Idris Abdullahi Nasir

Mobile:

$+2348022851352$

E mail: eedris888@yahoo.com

Key words: Antimalarial resistance; pregnant women; Malaria control
Background and study aim: Despite the global priority given to malaria control and prevention, antimalarial resistance is a major factor that encourages persistence of malaria in developing countries. This prospective study sought to determine the antimalarial resistance pattern of $P$. falciparum isolated from infected pregnant women attending antenatal clinics at Kaduna state, Northern Nigeria.

Materials and Methods: Between 16th February and 28th April, 2015, EDTA anticoagulated blood samples were collected from seventy nine pregnant women with plasmodiasis. Antimalarial susceptibility of chloroquine, artesunate, artemether and sulfadoxin-pyrimethamine (SP) against $P$ falciparum was done using schizont maturation assay. Multidrug resistant plasmodiasis was defined by resistance to $\geq 3$ antimalarial drugs.

Results: Malaria parasites from the pregnant women exhibited the highest resistance against chloroquine, $85(24.1 \%)$ followed by Artemether, 30 (8.5\%) then sulfadoxin-pyrimethamine, $29(8.2 \%)$ and least resistant to artesunate, $28(7.9 \%)$. The occurrence rate of multidrug resistance was $40.5 \%$. There was no significant association between occurrence of multidrug resistance and malaria parasitaemia ( $\mathrm{p}=0.092$ ). Seventy five, $94.8 \%$ of the $P$. falciparum infected subjects exhibit resistant to at least one antimalarial. Antimalarial resistance was highest in women with severe malaria 20 (80.0\%), followed by those with moderate malaria, $15(62.5 \%)$ and least in those with mild malaria, $4(13.3 \%)$. There was significant association between occurrence of antimalarial resistance and densities of malaria parasitaemia $(\mathrm{p}=0.0125)$.

Conclusion: Considering the high degree of antimalarial resistance reported from this study, there will be challenges in eradicating malaria in this environment. These findings necessitate the need for regular surveillance for resistant $P$. falciparum and evaluation of more effective alternative drug (s).

\section{INTRODUCTION}

Malaria is a major public health challenge in sub-Saharan Africa. In 2015, there were an estimated 214 million cases and 438,000 deaths due to malaria globally with Nigeria accounting for $25 \%$ of these [1]. Antimalarial resistance is a major factor that encourages persistence of malaria in developing countries. Despite the public health priority given to malaria, in recent time, the parasite has developed resistance to most of the commonly available prophylactic and therapeutic antimalarial agents $[\mathbf{1 , 2}]$.
In view of these, the need for periodic antimalarial sensitivity testing becomes crucial. The main objective of antimalarial sensitivity testing is to evaluate the parasite (s) sensitivity to increasing antimalarial dosages. This approach allows for complete exclusion of interfering host immunity and metabolism of the compound, thus offering an accurate evaluation of drug impact on parasites [3].

Over the past five decades, antimalarial resistance by Plasmodium falciparum has become an issue of utmost concern. In vitro assays for assessing antimalarial 
drug sensitivity have become indispensable tools for the surveillance of drug resistance and the planning of therapeutic guidelines. These include Schizont Maturation assay, radiolabeled hypoxanthine isotopic assay, malaria histidinerich protein 2 (HRP2) and lactate dehydrogenase (LDH) quantification by enzyme-linked immunosorbent assay (ELISA) and nucleic acid amplification tests such as polymerase chain reaction (PCR) [4].

The ideal approach for detecting and monitoring antimalarial resistance is the use of polymerase chain reaction. This method detects genes responsible for resistance and results are usually available within 8-12 hours accompanied with high sensitivity and specificity. However, the need for technical training of laboratory personnel (especially in developing countries) and its relatively high cost of procurement and maintenance limits PCR applicability in resource limited settings [4].

A key intervention for controlling malaria and its effects during pregnancy is the administration of intermittent preventive treatment (IPT) [5]. This consists of a complete therapeutic course of antimalarial medicine given to pregnant women at routine prenatal visits, regardless of whether they are infected with malaria or not. IPT reduces incidences of maternal malaria episodes as well as maternal and placental parasitaemia. Therefore, the WHO recommends IPT with SP in areas with stable malaria transmission in the subSaharan Africa [6].The efficacy of SP is being compromised in Africa by the emergence of several mutations which has been shown to reduce the efficacy of intermittent preventive treatment [7-9]. Importantly, the prevalence of resistance to SP should be determined. Accordingly, IPT-SP should be used in regions with SP resistance prevalence less than 30\% [9]. This prospective study sought to determine the antimalarial resistance pattern of $P$. falciparum isolated from infected pregnant women attending antenatal clinics at Kaduna state, Northern Nigeria.

\section{MATERIALSAND METHODS}

\section{Study Area}

This prospective study was conducted on pregnant women receiving antenatal services at General Hospital Kawo, Yusuf Dantsoho Hospital, Gwamna Awon Hospital and Barau Dikko Hospital, in Kaduna state, Northern Nigeria. The selection of health facilities was based on ease of accessibility and number of antenatal attendance, Kaduna State lies at latitude $10^{\circ} 20^{\prime}$ north and longitude $7^{\circ} 45^{\prime}$ east. It has a population of $6,113,503$ (2006 census figures) and a population density of 130 people per square kilometer.

\section{Sampling method, Inclusion and Exclusion criteria:}

Known pregnant women with malaria parasitaemia at any gestation age who reside within the study area were included; however, those with chronic debilitating diseases were excluded.

\section{Ethical clearance and informed consent:}

The study protocol was performed according to the Helsinki declaration and approved by ethical research committee of Kaduna State Ministry of Health, Kaduna State, Nigeria. Informed written consent was obtained from all subjects before recruited for the study.

Sample collection, preparation and precaution Between $16^{\text {th }}$ February and $28^{\text {th }}$ April, 2015, about $2 \mathrm{ml}$ of venous blood samples were collected aseptically from 79 individual subjects and carefully dispensed into EDTA sample containers.

\section{Malaria Microscopy:}

The parasite species were identified by preparing thick and thin blood films on microscope slides using the Giemsa staining techniques as was described by Cheesbrough [10]. Two glass slides were labeled for each participant. A drop of blood was then placed on the clean, grease free glass slide and allowed to dry. For thick films, a drop of blood was dropped at the center of a clean grease-free microscope slide to cover an area of $15 \mathrm{~mm}$ in diameter. The smears were allowed to air-dry after which they were covered with $10 \%$ Giemsa stain, then allowed to stay for 10 minutes before the stains were washed off with water. The thick films were used to detect the presence of malaria parasites in blood samples. Parasite species and morphology were determined by microscopic examination of the thin films which were also done with the $10 \%$ Giemsa stain for 5 minutes. The microscopic examination of the stained slides were done using the oil immersion objective lens (100x objective). At least 100 high power fields were examined before a thick smear was declared negative. Malaria parasites were counted per $500 \mathrm{WBC}$, which were used to estimate the parasite density per microlitre of blood [10]. 
Antimalarial drug susceptibility assay:

Schizont maturation inhibition technique was used. Chloroquine, artesunate, artether and sulfadoxinpyrimethamine were assessed for their in-vitro antimicrobial actions on the $P$. falciparum isolated. These drugs were purchased from the shelf (pharmacy store). Stock solutions of the antimalarial drugs will be prepared as follows:

The drugs were made to be of equal concentrations of $100 \mathrm{mg} / \mathrm{ml}$ by dissolving in the appropriate amount of distilled water. 50mg (1 tablet) of artesunate was dissolved in $0.5 \mathrm{ml}$ of distilled water, $250 \mathrm{mg}$ of chloroquine was dissolved in $2.5 \mathrm{ml}$ of distilled water, $250 \mathrm{ml}$ of artemeter in $750 \mathrm{ml}$ of distilled water. They were allowed to dissolve and the suspensions were shaken thoroughly to get homogenous solutions. Twofold serial dilutions of each of the drug suspension was prepared by adding $1 \mathrm{ml}$ of the suspension into $1 \mathrm{ml}$ of distilled water in the first test tube, from the first tube, $1 \mathrm{ml}$ of the solution was taken and added into the second tube already containing $1 \mathrm{ml}$ of distilled water. This was done to the fifth tube for all the drugs. Malaria infected blood samples were centrifuged at 2000 revolutions per minute $(2000 \mathrm{rpm})$ for five minutes. After centrifugation, the plasma and buffy coats (leukocyte interface) discarded and $0.2 \mathrm{ml}$ of the packed red blood cells was dispensed into thirteen test tubes for each of the samples. The tubes contained different concentrations of the drugs in duplicates. A control (tube without drugs) was set up for each of the samples. $1 \mathrm{ml}$ of the physiological saline was dispensed into each of the tubes. After addition, lids were placed over the tubes and the tubes shaken gently to dissolve the drugs.

The samples were then incubated at $37^{\circ} \mathrm{C}$ for 42 hours. At the end of incubation, thick and thin blood films were prepared from the samples in each tube including those of the controls. The number of schizonts or gametocytes in the control tubes was compared with that in the other tubes containing different concentrations of the antimalarial drugs.

\section{Statistical analysis}

Results were presented in tabular forms showing the frequencies and percentages of antimalarial resistance in each drug tested. Data were analyzed for statistical association between antimalarial resistance and the densities of malaria parasitaemia in pregnant women using two tailed Chi-square test.

\section{RESULTS}

In total, 79 malaria infected pregnant women in their various pregnancy trimesters were recruited for the study, pregnant women ages were between 17 and 44 years and mean age was $22.45 \pm 6.84$ $( \pm \mathrm{SD})$. All the malaria positive cases were that of Plasmodium falciparum. Malaria parasites from pregnant women exhibited the highest resistance against chloroquine, $75(94.9 \%)$ and least resistant to artesunate, 28 (35.4\%). The occurrence rate of multidrug resistance is $40.5 \%$. There was no significant association between occurrence of multidrug resistance and malaria parasitaemia in pregnant women $(\mathrm{p}=0.092)$ (Table 1). Seventy five, $94.8 \%$ of the $P$. falciparum infected subjects were resistant to at least one antimalarial. Antimalarial resistance was highest in women with severe malaria 25 (100\%), followed by those with moderate malaria, $23(95.8 \%)$ and least in those with mild malaria, $27(90.0 \%)$. There was significant association between occurrence of antimalarial resistance and densities of malaria parasitaemia $(\mathrm{p}=0.0125)$ (Table 2$)$.

Table (1): In-vitro antimalarial resistance pattern in pregnant women infected with malaria

\begin{tabular}{|l|l|c|c|c|}
\hline S/No. & \multicolumn{1}{|c|}{ Antimalarial } & No. (\%) resistant & No. (\%) susceptible & $P$-value \\
\hline 1 & Artemether & $30(38.0)$ & $49(62.0)$ & \\
\hline 2 & Chloroquine & $75(94.9)$ & $4(5.1)$ & \\
\hline 3 & Artesunate & $28(35.4)$ & $51(64.6)$ & \\
\hline 4 & Sulfadoxine-pyrimethamine & $29(36.7)$ & $50(63.3)$ & \\
\hline 5 & $\begin{array}{l}\text { Resistant to } \geq 3 \text { antimalarial } \\
\text { drugs (MDR) }\end{array}$ & $32(40.5)$ & $47(59.5)$ & $0.092^{*}$ \\
\hline
\end{tabular}

*Significant association determined by Chi-square test 
Table (2): Distribution of antimalarial resistance by malaria densities among pregnant women

\begin{tabular}{|l|c|c|c|c|c|}
\hline & $\begin{array}{c}\text { Mild Malaria } \\
\text { Parasitaemia } \\
(<\mathbf{1 0 0 0} \\
\text { parasites/uL of } \\
\text { Blood) }(\mathbf{\%})\end{array}$ & $\begin{array}{c}\text { Moderate Malaria } \\
\text { Parasitaemia } \\
\mathbf{( 1 0 0 0} \mathbf{- 1 0 , 0 0 0} \\
\text { parasites/uL of } \\
\text { Blood) }(\boldsymbol{\%})\end{array}$ & $\begin{array}{c}\text { Severe Malaria } \\
\text { Parasitaemia } \\
(>\mathbf{1 0 0 0 0} \\
\text { parasites/uL of } \\
\text { Blood) }(\mathbf{\%})\end{array}$ & Total & $\begin{array}{c}\text { P } \\
\text { Value }\end{array}$ \\
\hline $\begin{array}{l}\text { No. of } \\
\text { subjects }\end{array}$ & 30 & 24 & 25 & 79 & \\
\hline $\begin{array}{l}\text { No. with } \\
\text { antimalarial } \\
\text { resistance }\end{array}$ & $(90.0)$ & 23 & 25 & 75 & $0.0125^{*}$ \\
\hline
\end{tabular}

*Significant association determined by Chi-square test

\section{DISCUSSION}

This study of the blood samples of malaria infected pregnant women showed that $94.8 \%$ of them attending antenatal clinics of secondary healthcare facilities had resistance to at least one antimalarial drug tested. Observation from this study also showed $5.2 \%$ (4/79) were sensitive in vitro to Chloroquine. This observation is similar to Folarin et al.[11] report that $85 \%(71 / 84)$ of malaria parasites in Ibadan, southwestern Nigeriawere resistant to Chloroquine. However, the result contradicts Balogun et al.[12] who reported that Plasmodium falciparum isolates in Northeastern Nigeria were more sensitive to Chloroquine. It was found that $51(64.6 \%)$ of the Plasmodium falciparum isolates were sensitive to Artesunate. This is similar to the study by $\mathrm{Na}-$ Bangchang et al. [13] who reported $36.7 \%$ declining in sensitivity to artesunate in ThaiMyanmar border. One of the factors to be considered in the prophylaxis, treatment, and control of Plasmodium falciparum malaria is the resistance of parasite strains that may arise against virtually every drug available. Identification of Pfcrt is the central determinant of chloroquine-resistant $P$. falciparum malaria that provides molecular marker that can be used for surveillance of resistance and to evaluate drug treatment and prophylaxis policies [14]. These findings also agreed with earlier findings where molecular markers of resistance were found in samples that gave in vivo resistance/ drug failure [11,14].

The presence of high resistance rate of $P$. falciparum isolates to SP is worrisome. Considering its universal acceptance and usage in the prevention of maternal malaria, in future, the public health benefits of IPT will probably decline due to SP resistance. Intensifying the use of ITNs, research on vaccine development and alternative drugs for IPT become priorities in prevention of malaria. A similar finding was reported by Bouyou-Akotet et al. [15].

In regards to monitoring antimalarial effectiveness in order establishment of the baseline sensitivity of malaria to commonly prescribed antimalarial,in-vitro drug sensitivity is important [15]. In-vitro resistance against chloroquine, arthether and artesunate was observed among the malaria infected pregnant women in this study. These results conform to earlier findings of Fallet al.[16]. Similarly, previous malaria resistance against chloroquine and arthemisinbased antimalarial agents has been reported by in Nigeria[14,17]. The host cells response to antimalarial drugs are controlled by the pharmacokinetic properties of drugs, preformed immunity in the patient, as well as the complexity of infections in high transmission areas [18]. These factors may contribute to the range of variations in the clinical expression of antimalarial resistance patterns.

\section{CONCLUSION}

This study showed the presence of high degree of antimalarial resistance by $P$. falciparum infections among pregnant women in Kaduna state, Nigeria. Based on the findings, there will be challenges in eradicating malaria in this environment. There is a need for periodic surveillance using molecular assays in order to identify the genes responsible for antimalarial resistance. In addition, it's recommended that evaluation of more effective and alternative antimalarial drug (s) be considered. 


\section{Acknowledgement}

We are grateful to $\mathrm{Mr}$ Abdurrahman Elfulaty Ahmad for providing technical input for this study.

\section{Conflict of Interest:}

\section{None}

\section{REFERENCES}

1. World Health Organization. World malaria report. 2015a; http://www.who.int/malaria/ publications/ world-malaria-report-2015/wmr2015-profiles. pdf [Accessed on 4th August, 2016].

2. Alibu VD, Egwang TG. Genomics Research and Malaria Control: Great Expectations. PLoS Biol 2003; 1(2): 39.

3. World Health Organization. The health of the people: what works-the African Regional Health Report 2014. http://apps.who.int/iris/bitstream/ 10665/137377/4/9789290232612.pdf [Accessed on 24th July, 2016]

4. World Health Organization. World Malaria Report 2015b. http://www.who.int/malaria/ publications/world-malaria-report-

2015/report/en/ [Accessed on 2nd August, 2016]

5. World Health Organization Evidence Review Group. Intermittent preventive treatment of malaria in pregnancy (IPTp) with sulfadoxine-pyrimethamine (SP). Geneva: World Health Organization; 2012. $1-17$.

6. World Health Organization. A strategic frameworkfor malaria prevention and control during pregnancy in the African region. Brazzaville: World Health Organization, Regional Office for Africa; 2004.

7. Gesase S, Gosling RD, Hashim R, Ord R, Naidoo I, Madebe R, et al. High resistance of Plasmodium falciparum to sulphadoxine/pyrimethamine in northern Tanzania and the emergence of dhps resistance mutation at Codon 581. PLoS One. 2009; 4(2): e4569

8. Minja DT, Schmiegelow C, Mmbando B, Bostrom S, Oesterholt M, Magistrado P, et al. Plasmodium falciparum mutant haplotype infection during pregnancy associated with reduced birthweight, Tanzania. Emerg. Infect. Dis. 2013;19(9): 1446-1454.

9. Naidoo I, Roper C. Mapping 'partially resistant', fully resistant' and 'super resistant' malaria. Trends Parasitol. 2013; 29(10):505-515.
10. Cheesbrough M. District Laboratory practices for Tropical Countries. 2005; 1: 120-124.

11. Folarin OA, Gbotosho GO, Sowunmi A, Olorunsogo OO, Oduola AM, Happi C. Chloroquine Resistant Plasmodium falciparum in Nigeria: Relationship between pfcrt and pfmdr1 Polymorphisms, InVitro Resistance and Treatment Outcome. Open Trop Med J. 2008; 1: 74-82.

12. Balogun ST, SandabeUK, WaziriIA, Jibrin J, Fehintola FA. In vitro sensitivity of Plasmodium falciparum clinical isolates to 4-aminoquinolines in Northeast Nigeria. MWJ. 2016; 7:10.

13. Na-Bangchang K, Muhamad P, Ruaengweerayut $\mathrm{R}$, Chaijaroenkul W, Karbwang J. Identification of resistance of Plasmodium falciparum to artesunate-mefloquine combination in an area along the Thai-Myanmar border: integration of clinico-parasitological response, systemic drug exposure, and in vitro parasite sensitivity. Malaria J. 2013; 12: 263-274.

14. Olasehinde GI, Ojurongbe OO, Fagade EO, Ruchi S, Egwari LO, Ajayi AA, et al. Detection of Molecular Markers of Antimalarial Drug Resistance in Plasmodium Falciparum from South-Western Nigeria. Covenant J Phy Life Sci.2014; 1 (2): 61-75.

15. Bouyou-Akotet MK, Mawili-Mboumba DP, Tchantchou TD, Kombila M. High prevalence of sulfadoxine/pyrimethamine-resistant alleles of Plasmodium falciparum isolates in pregnant women at the time of introduction of intermittent preventive treatment with sulfadoxine/pyrimethamine in Gabon. $J$ Antimicrob Chemother 2010; 65: 438-441.

16. Fall B, Madamet M, Camara C, Amalvict R, Fall M, Nakoulima A, et al. Plasmodium falciparum In Vitro Resistance to Monodesethylamodiaquine, Dakar, Senegal, 2014. Emerg Infect Dis 2016; 22 (5): 841-845.

17. Happi TC, Thomas SM, Gbotosho GO, Falade CO, Akinboye DO, Gerena L, et al. Point mutations in the pfert and pfmdr-1 genes of Plasmodium falciparum and clinical response to chloroquine, among malaria patients from Nigeria. Ann Trop Med Parasitol 2003; 97: 439-451.

18. Ibrahim ML, Gay-Andrieu F, Adehossi E, Lacroix V, Randrianarivelojosia M, Duchemin JB. Fieldbased evidence for the linkage of pfcrt and pfdhfr drug-resistant malaria genotypes and clinical profiles for severe malaria in Niger. Microb Infect 2007; 9: 599-604. 